\title{
Impact of disease progression on individual IPSS trajectories and consequences of immediate versus delayed start of treatment in patients with moderate or severe LUTS associated with BPH
}

\author{
Salvatore D'Agate ${ }^{1} \cdot$ Timothy Wilson $^{2} \cdot$ Burkay Adalig $^{3} \cdot$ Michael Manyak $^{4} \cdot$ Juan Manuel Palacios-Moreno $^{5}$. \\ Chandrashekhar Chavan ${ }^{6} \cdot$ Matthias Oelke $^{7}$. Claus Roehrborn ${ }^{8} \cdot$ Oscar Della Pasqua $^{1,9}$ (i)
}

Received: 5 December 2018 / Accepted: 23 April 2019 / Published online: 11 May 2019

(c) The Author(s) 2019

\begin{abstract}
Purpose Despite superiority of tamsulosin-dutasteride combination therapy versus monotherapy for lower urinary tract symptoms due to benign prostatic hyperplasia (LUTS/BPH), patients at risk of disease progression are often initiated on $\alpha$-blockers. This study evaluated the impact of initiating tamsulosin monotherapy prior to switching to tamsulosin-dutasteride combination therapy versus immediate combination therapy using a longitudinal model describing International Prostate Symptom Score (IPSS) trajectories in moderate/severe LUTS/BPH patients at risk of disease progression.

Methods Clinical trial simulations (CTS) were performed using data from 10,238 patients from Phase III/IV dutasteride trials. The effect of varying disease progression rates was explored by comparing profiles on- and off-treatment. CTS scenarios were investigated, including a reference (immediate combination therapy) and six alternative virtual treatment arms (delayed combination therapy of 1-24 months). Clinical response ( $\geq 25 \%$ IPSS reduction relative to baseline) was analysed using log-rank test. Differences in IPSS relative to baseline at various on-treatment time points were assessed by $t$ tests.

Results Delayed combination therapy initiation led to significant $(p<0.01)$ decreases in clinical response. At month 48 , clinical response rate was $79.7 \%$ versus $74.1 \%, 70.3 \%$ and $71.0 \%$ and IPSS was 6.3 versus 7.6, 8.1 and 8.0 (switchers from tamsulosin monotherapy after 6, 12 and 24 months, respectively) with immediate combination therapy. More patients transitioned from severe/moderate to mild severity scores by month 48 .

Conclusions CTS allows systematic evaluation of immediate versus delayed combination therapy. Immediate response to $\alpha$-blockers is not predictive of long-term symptom improvement. Observed IPSS differences between immediate and delayed combination therapy (6-24 months) are statistically significant.
\end{abstract}

Keywords Benign prostatic hyperplasia $\cdot$ Clinical trial simulation $\cdot$ Drug-disease modelling $\cdot$ Dutasteride $\cdot$ Lower urinary tract symptoms · Tamsulosin

Electronic supplementary material The online version of this article (https://doi.org/10.1007/s00345-019-02783-x) contains supplementary material, which is available to authorized users.

Oscar Della Pasqua

oscar.e.della-pasqua@gsk.com

1 Clinical Pharmacology and Therapeutics Group, University College London, London, UK

2 PAREXEL International, Durham, NC, USA

3 Classic and Established Products, GSK, Istanbul, Turkey

4 Classic and Established Products, GSK, Washington, DC, USA

5 Classic and Established Products, GSK, Madrid, Spain
6 Classic and Established Products, GSK, Worli, Mumbai, India

7 Department of Urology, St. Antonius Hospital, Gronau, Germany

8 Department of Urology, University of Texas Southwestern Medical Center, Dallas, TX, USA

9 Clinical Pharmacology Modelling and Simulation, GSK, Stockley Park, 1-3 Ironbridge Road, Uxbridge, Middlesex UB11 1BT, UK 


\section{Introduction}

The primary treatment goal for lower urinary tract symptoms associated with benign prostatic hyperplasia (LUTS/ $\mathrm{BPH})$ is to reduce bothersome symptoms, with clinical management now focused on altering disease progression and preventing long-term complications such as surgery or acute urinary retention (AUR) [1-3]. Treatment guidelines for patients at risk of progression centre on two drug classes: $5 \alpha$-reductase inhibitors (5-ARI) and $\alpha$-blockers [4]. These recommendations are supported by data from large clinical studies, which indicate a significant reduction in risk of AUR and BPH-related surgery in patients treated with combination therapy [5, 6]. Additionally, the 4-year Combination of Avodart and Tamsulosin (CombAT) study showed that the dutasteride-tamsulosin combination was more effective than tamsulosin monotherapy in reducing the relative risk of AUR, BPH-related surgery, and BPH clinical progression in men with moderate-tosevere LUTS at increased risk of progression [7].

Following a LUTS/BPH diagnosis, patients are often treated initially with an $\alpha$-blocker rather than combination therapy, including those at risk of disease progression. Whilst $\alpha$-blockers may provide immediate symptomatic improvement, it is not possible to predict which patients will show sustained response over time, even if higher baseline symptoms [8] and/or larger prostates [9] are prognostic for deterioration of symptoms. No baseline or clinical factors have yet been identified that are sufficiently sensitive and specific to discriminate progression risk from long-term treatment response in individual patients [10]. Delaying combination therapy may reduce long-term benefits due to the absence of a disease-modifying moiety in the initial treatment phase.

The implications of deviating from clinical guidelines about the use of combination therapy may have been overlooked. Currently, treatment response is mostly assessed in clinical trials and clinical practice by the absolute change from baseline on the International Prostate Symptom Score (IPSS) compared to placebo or active therapy. However, this does not provide insight into the effect of treatment on the rate of progression over time [11, 12]; rather, it describes the magnitude of change over a predefined interval or time span. With respect to the profile of the IPSS measurements over time, any attempt to linearise or interpolate underlying progression rates from changes observed between consecutive visits may lead to inaccurate estimates of disease progression [11].

Recently, we developed a longitudinal model using randomised clinical trial data and follow-up studies including placebo, tamsulosin, dutasteride and combination therapy, which enabled the characterisation of individual
IPSS trajectories or profiles [13]. This model provides an opportunity to evaluate the impact of different interventions, taking into account the role of other covariate factors known to affect response in patients with LUTS/BPH with moderate or severe symptoms.

Here, we use computer simulations in conjunction with baseline characteristics from patients participating in previous clinical trials to characterise the deterioration of symptoms associated with varying rates of disease progression and evaluate the long-term effects of different interventions based on individual IPSS trajectories. More specifically, we aim to assess the implications of delayed start of combination therapy in patients with moderate and severe symptoms.

\section{Patients and methods}

Initially, simulations were performed using the longitudinal model recently developed by D'Agate et al. [13] to illustrate the implications for treatment response when drugs with disease-modifying properties are used in patients with varying rates of disease progression. Further details of the model, including a description of the parameters and its implementation for the initial set of simulations are presented in the Supplemental Materials. Based on this preliminary evaluation, baseline IPSS and disease progression rates interact with treatment effect, making it difficult to disentangle the contribution of each factor to the overall response at the end of a predefined interval, as assessed in most clinical trials. These findings support the use of clinical trial simulations (CTS) based on individual IPSS trajectories to assess the deterioration of symptoms associated with varying rates of disease progression and evaluate the effect of early versus delayed onset of combination therapy with the tamsulosin and dutasteride.

Different CTS scenarios were evaluated, taking into account demographic and clinical covariates known to affect response. The selected scenarios provide insight into the magnitude of treatment response differences following the initiation of combination therapy at different time points. An overview of the CTS steps, including the main protocol design characteristics, data input and output, is presented in Fig. 1a.

\section{Data source}

Demographic and clinical characteristics of the patient pool $(N=10,238)$ included in the CTS were obtained from six Phase III/IV clinical trials (ARIA3001, ARIA3002, ARI40002, CombAT, CONDUCT, ARIB3003). An overview of the patient population is shown in Supplementary Table S1. In CONDUCT, tamsulosin and dutasteride were 
administered as a fixed-dose combination, while other studies administered treatment as separate dosage forms.

\section{CTS scenarios}

These evaluated the impact of immediate versus delayed start of combination therapy, and focused on the assessment of treatment response defined as the proportion of subjects with a drop in IPSS $\geq 25 \%$ from baseline (i.e., the same endpoint in CombAT [14] and CONDUCT [15] studies included in this analysis) to signify a clinically meaningful improvement (CMI) at months 12, 24, 36 and 48. Additionally, the relative difference in the proportion of patients who showed a CMI and transitioned between severity categories was evaluated at months 1, 3, 6, 12, 24 and 48, as described below:

1. Severely symptomatic (IPSS 20-35) to mildly symptomatic (IPSS 1-7).

2. Moderately symptomatic (IPSS 8-19) to mildly symptomatic (IPSS 1-7).
All CTS were performed assuming a protocol design aimed at mimicking clinical guidelines, in which nonresponders to $\alpha$-blockers (patients who had a change in IPSS $<25 \%$ of baseline, i.e. who did not experience a CMI) switched to combination therapy at the selected time points. Given the large population sample size included in each scenario, ten replicate trials were considered sufficient to calculate the $90 \%$ confidence intervals (CIs) of the estimates. For clarity, graphical summaries were created using a single replicate trial. Full details of the protocol design characteristics are shown in Table $\mathrm{S} 2$.

\section{Results}

\section{Effect of immediate versus delayed start of combination therapy}

Demographic characteristics of patients enrolled into each virtual treatment arm are summarised in Table S3. Figure $1 \mathrm{~b}$ shows the mean IPSS change from baseline and predicted response rate for each treatment scenario. The different curves indicate that switching from tamsulosin monotherapy to combination therapy $\geq 6$ months from the

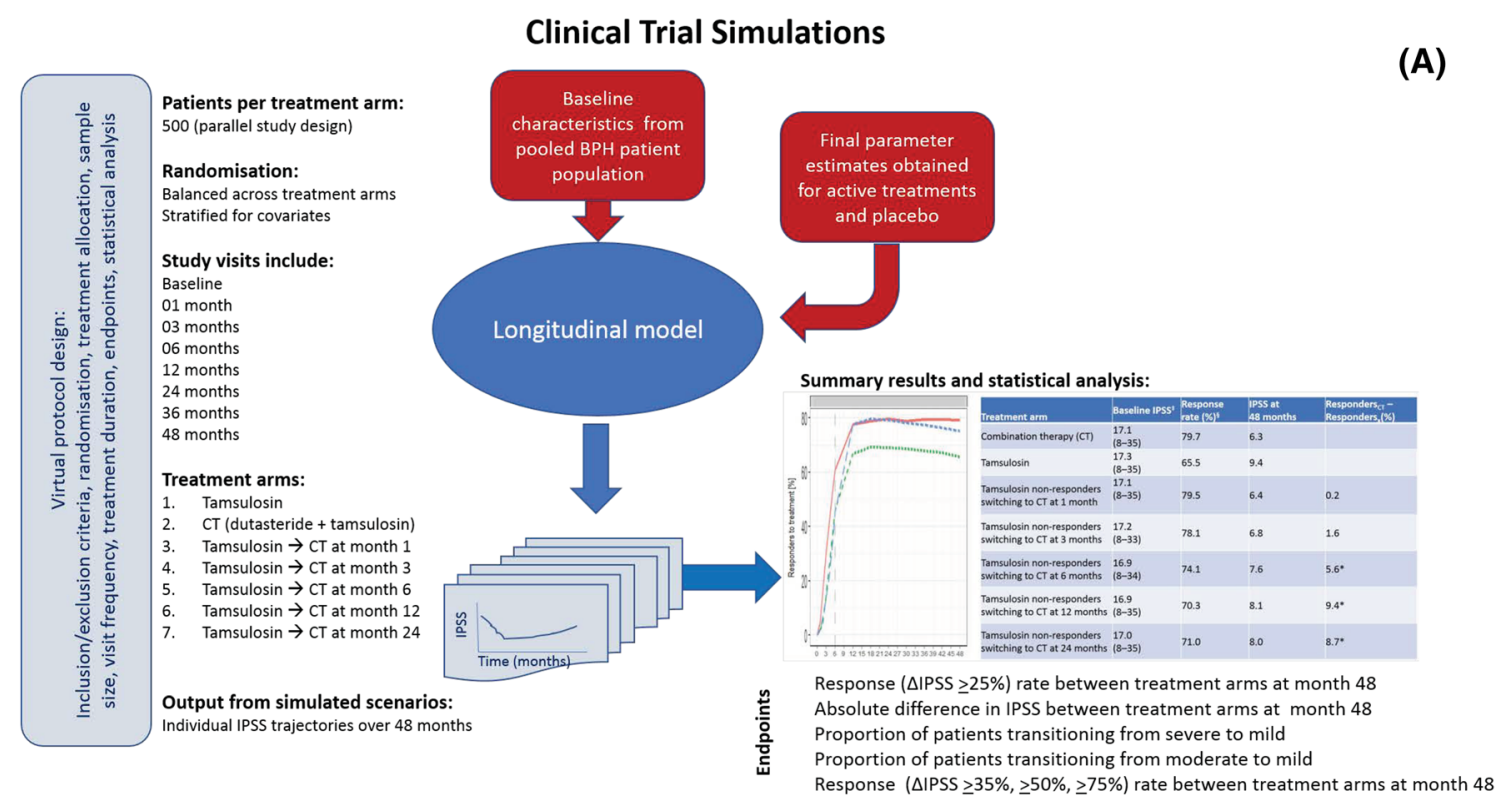

Fig. 1 a Schematic diagram of CTS based on the longitudinal model describing individual IPSS trajectories [13]. b Mean IPSS changes from baseline (left panels) and predicted response rate (\% responders) (right panels) stratified by treatment scenario according to a parallelstudy design. From top to bottom, each panel depicts the predicted profiles for tamsulosin monotherapy (green dotted line), tamsulosindutasteride combination therapy (red solid line), and treatment arm switching to combination therapy (blue dashed line) at 1, 3, 6, 12 and 24 months. The lines depicting tamsulosin monotherapy and tamsulosin-dutasteride combination therapy are constant and represent these treatments being applied throughout 48 months. The dashed vertical lines indicate time of switch to combination therapy. Graphical summaries and statistical analysis refer to the results of a single replicate trial. In these simulations, the placebo effect was assumed to occur only immediately after enrolment into the study. Placebo effect is a key component of the initial response and can last longer than 6 months, as assessed by its half-life. No studies included a placebo treatment arm for $>2$ years, so it was not possible to establish whether inter-individual differences might allow for a longer placebo effect 
(B)

Mean IPSS change from baseline
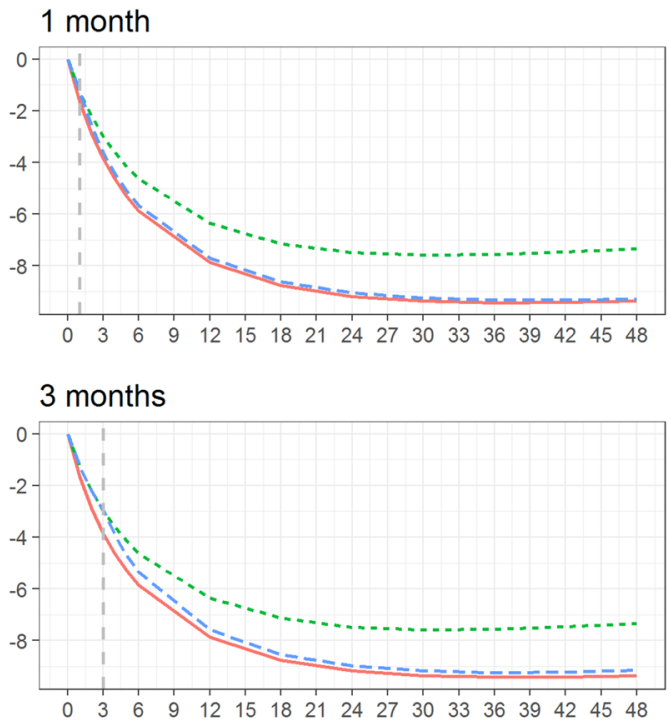

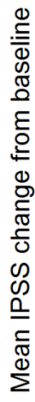
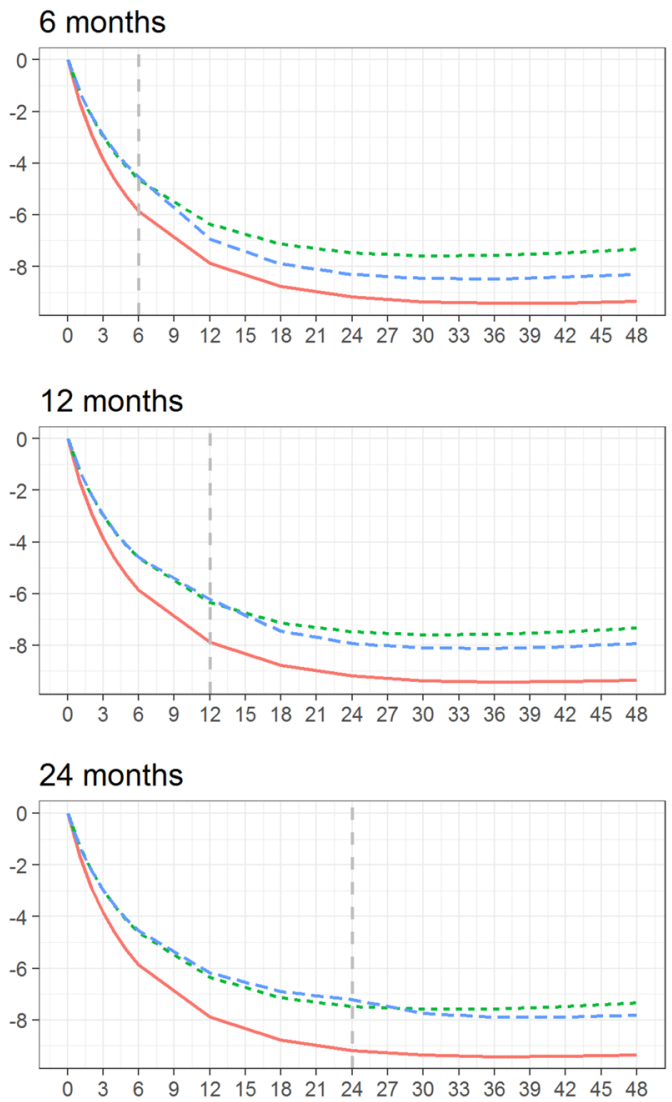

Time [months]
Predicted response rate (\% responders)
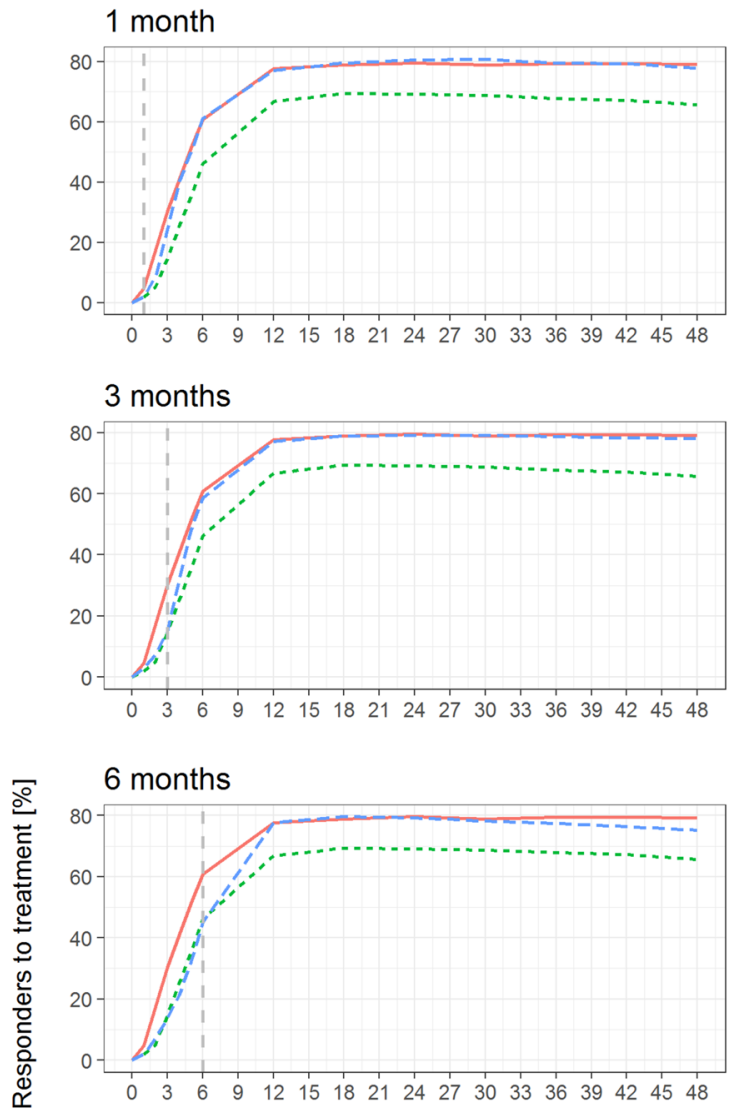

\section{2 months}

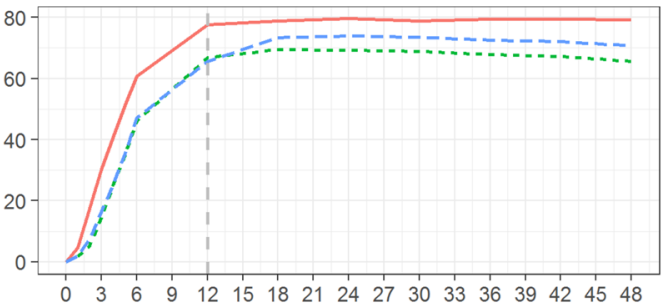

\section{4 months}

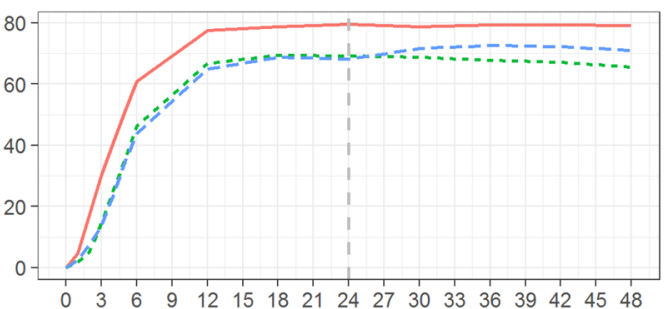

Time [months]

Fig. 1 (continued) 


\begin{tabular}{|c|c|c|c|c|}
\hline Treatment scenario & $\begin{array}{l}\triangle I P S S \text { at } \\
1 \text { year* }\end{array}$ & $\begin{array}{l}\triangle I P S S \text { at } \\
2 \text { years* }\end{array}$ & $\begin{array}{l}\triangle I P S S \text { at } \\
3 \text { years* }\end{array}$ & $\begin{array}{l}\triangle I P S S \text { at } \\
4 \text { years* }\end{array}$ \\
\hline Tamsulosin non-responders to CT at 1 month & 1 & 1 & 1 & 1 \\
\hline Tamsulosin non-responders to CT at 3 months & 1 & 1 & 1 & 1 \\
\hline Tamsulosin non-responders to CT at 6 months & 0.168 & 1 & 1 & 0.704 \\
\hline Tamsulosin non-responders to CT at 12 months & $<0.001$ & 0.098 & 0.127 & 0.081 \\
\hline Tamsulosin non-responders to CT at 24 months & $<0.001$ & $<0.001$ & 0.022 & 0.038 \\
\hline \multicolumn{5}{|c|}{$\begin{array}{l}\text { *Bonferroni corrected p-values for the change from baseline in mean IPSS of the different treatment } \\
\text { scenarios, compared with reference (i.e. combination therapy (CT)). The change from baseline in mean IPSS } \\
\text { up to month } 48 \text { was a secondary endpoint in the clinical trial simulation and as such, the study was not } \\
\text { powered to detect a pre-defined difference between treatment arms for this endpoint. }\end{array}$} \\
\hline
\end{tabular}

Fig. 1 (continued)

start of treatment has a statistically significant effect on the proportion of responders at month 48 .

The CTS scenarios confirm the anticipated impact of delaying combination therapy, with the observed differences in IPSS at month 48 appearing to be determined by the delay. The total number of subjects transitioning to combination therapy at the different visits is summarised in Table 1 (panels $\mathrm{A}$ and $\mathrm{B}$ ), along with responder rate at end of the study and $90 \%$ CIs from ten trial replicates. From 6 months onwards, a significantly lower proportion of responders was observed in each virtual treatment arm relative to the combination therapy arm. These results are complemented by median estimates of the response rate for a single trial replicate (Table S4). Baseline characteristics of the patients in each treatment arm are summarised to highlight that no other factor appears to predict the lack of response or explain which patients switch from tamsulosin monotherapy after 6,12 or 24 months.

As summarised in Table 1 (panels $C$ and D), the impact of the delayed start of combination treatment is also reflected in the total number of patients who transition from severe or moderate to mild IPSS categories over the 48-month period. In addition, the lower panel in Table 1 provides further evidence of the difference in magnitude of clinical improvement, assessed by percentage of responders per treatment arm with a decrease in IPSS $\geq 25 \%, \geq 35 \%, \geq 50 \%$ or $\geq 75 \%$ relative to baseline. These results indicate that a significant proportion of patients showed greater improvement in symptoms when combination therapy was started immediately.

\section{Discussion}

Meta-analyses have been used to compare different treatment options. However, this technique allows scrutiny only of design factors that have been implemented, without necessarily correcting for the effect of confounding factors which cannot be easily excluded. Moreover, they often focus on mean parameter estimates, yielding results that ignore underlying covariates that may modify the treatment effect. By contrast, the application of longitudinal modelling and CTS at individual patient level allows investigation of a range of design characteristics on the power to detect treatment effects, without confounding or practical restrictions, prior to exposing patients to an experimental intervention [16, 17]. CTS can be performed not only to evaluate scenarios that have not been previously investigated in clinical trials, but also to explore hypothetical scenarios which cannot be implemented in real-life conditions. Indeed, the implementation of a prospective, controlled study in which combination therapy is delayed may be ethically questionable, especially when guidelines recommend it in patients considered at risk for progression of BPH [4]. Here we have shown how this methodology can be used to explore design factors, such as delayed start of treatment, whilst disentangling it from other factors and interactions. Our analysis also provided an opportunity to assess the effect of disease progression, baseline covariates, and drug treatment on individual IPSS trajectories. 
Table 1 CTS results for each treatment arm based on a parallel-study design (ten trial replicates)

\begin{tabular}{|c|c|c|c|c|}
\hline \multicolumn{3}{|l|}{ (A) Treatment duration } & \multicolumn{2}{|c|}{$\begin{array}{l}\text { Median number of patients transition- } \\
\text { ing to combination therapy }(90 \% \mathrm{CI})\end{array}$} \\
\hline Start of treatment & & & 0 & \\
\hline 01 month & & & $489(487,493)$ & \\
\hline 03 months & & & $428(413,444)$ & \\
\hline 06 months & & & $284(266,306)$ & \\
\hline 12 months & & & $174(163,188)$ & \\
\hline 24 months & & & $157(153,173)$ & \\
\hline 36 months & & & 0 & \\
\hline 48 months & & & 0 & \\
\hline (B) Treatment arm & Baseline IPSS $^{\mathrm{a}}$ & Response rate $(\%)^{\mathrm{b}}$ & IPSS at 48 months & $\begin{array}{l}\text { Responders }_{\mathrm{CT}}^{-} \\
\text {Responders }_{x}(\%)\end{array}$ \\
\hline Combination therapy (CT) & $17.1[8-35]$ & $79.7(78.6,81.2)$ & $6.3(6,6.7)$ & \\
\hline Tamsulosin & $17.3[8-35]$ & $65.5(63.1,68.1)$ & $9.4(8.8,9.9)$ & \\
\hline Tamsulosin non-responders to $\mathrm{CT}$ at 1 month & $17.1[8-35]$ & $79.5(76.6,81.4)$ & $6.4(6.2,6.6)$ & 0.2 \\
\hline Tamsulosin non-responders to CT at 3 months & $17.2[8-33]$ & $78.1(76.8,81.0)$ & $6.8(6.4,7.1)$ & 1.6 \\
\hline Tamsulosin non-responders to $\mathrm{CT}$ at 6 months & $16.9[8-34]$ & $74.1(72.3,75.5)$ & $7.6(7.2,8.1)$ & $5.6^{*}$ \\
\hline Tamsulosin non-responders to CT at 12 months & $16.9[8-35]$ & $70.3(67.3,72.1)$ & $8.1(7.5,8.6)$ & $9.4^{*}$ \\
\hline Tamsulosin non-responders to CT at 24 months & $17.0[8-35]$ & $71.0(68.6,72.1)$ & $8.0(7.8,8.3)$ & $8.7^{*}$ \\
\hline
\end{tabular}

(C) Treatment arm Transition from moderate to mild at different study visits, cumulative \% (range)

\begin{tabular}{lllllll} 
& 1 month & 3 months & 6 months & 12 months & 24 months & 48 months \\
\hline Combination therapy (CT) & 4.6 & 12.3 & 22.3 & 35.3 & 44.7 & 45.5 \\
& $(3.8,5.2)$ & $(11.2,13.8)$ & $(20.5,23.3)$ & $(34.4,37.0)$ & $(43.4,46.4)$ & $(44.2,46.6)$ \\
Tamsulosin & 3.0 & 8.0 & 13.6 & 25.2 & 32.9 & 32 \\
& $(2.8,3.42)$ & $(6.5,8.4)$ & $(13.4,14.6)$ & $(23.4,26.1)$ & $(32.0,34.8)$ & $(31.0,34.2)$ \\
Tamsulosin non-responders & 4.1 & 11.0 & 21.6 & 35.1 & 43.3 & 43 \\
switching to CT at 1 month & $(3.6,5)$ & $(10.6,12.2)$ & $(20.6,23.0)$ & $(34.4,37.3)$ & $(41.7,45.8)$ & $(41.4,46.1)$ \\
Tamsulosin non-responders & 3.9 & 7.7 & 17.3 & 33.0 & 41.6 & 40.7 \\
switching to CT at 3 months & $(3.29,4.4)$ & $(6.3,9.2)$ & $(15.9,21.0)$ & $(31.1,36.1)$ & $(39.2,44.6)$ & $(38.3,44.0)$ \\
Tamsulosin non-responders & 3.2 & 7.6 & 14.4 & 30.5 & 40.7 & 40.4 \\
switching to CT at 6 months & $(2.6,3.5)$ & $(7.0,8.9)$ & $(12.8,15.8)$ & $(29.1,33.6)$ & $(38.6,42.6)$ & $(37.9,42.5)$ \\
Tamsulosin non-responders & 3.6 & 8.4 & 14.8 & 25.2 & 37.9 & 37.2 \\
switching to CT at 12 months & $(3.4,4.2)$ & $(7.4,9.4)$ & $(13.2,15.9)$ & $(23.0,27.5)$ & $(35.9,40.9)$ & $(35.7,40.7)$ \\
Tamsulosin non-responders & 3.3 & 8.1 & 14.7 & 25.4 & 34.3 & 38.9 \\
switching to CT at 24 months & $(3.0,4.2)$ & $(7.0,9.6)$ & $(12.7,16.4)$ & $(23.1,27.0)$ & $(32.7,35.0)$ & $(37.0,40.6)$ \\
\hline
\end{tabular}

(C) Treatment arm Transition from severe to mild at different study visits, cumulative \% (range)

\begin{tabular}{|c|c|c|c|c|c|c|}
\hline & 1 month & 3 months & 6 months & 12 months & 24 months & 48 months \\
\hline Combination therapy (CT) & $\begin{array}{l}0 \\
(0,0.1)\end{array}$ & $\begin{array}{l}0.4 \\
(0.2,0.8)\end{array}$ & $\begin{array}{l}2.2 \\
(1.6,2.8)\end{array}$ & $\begin{array}{l}6.6 \\
(4.8,7.1)\end{array}$ & $\begin{array}{l}12.8 \\
(11.5,13.4)\end{array}$ & $\begin{array}{l}15.7 \\
(13.8,16.4)\end{array}$ \\
\hline Tamsulosin & $\begin{array}{l}0 \\
(0,0)\end{array}$ & $\begin{array}{l}0.2 \\
(0,0.7)\end{array}$ & $\begin{array}{l}1.1 \\
(0.8,1.5)\end{array}$ & $\begin{array}{l}2.8 \\
(2.6,4.3)\end{array}$ & $\begin{array}{l}6.8 \\
(6.1,9.0)\end{array}$ & $\begin{array}{l}10.2 \\
(9.0,12.2)\end{array}$ \\
\hline $\begin{array}{l}\text { Tamsulosin non-responders } \\
\text { switching to CT at } 1 \text { month }\end{array}$ & $\begin{array}{l}0 \\
(0,0)\end{array}$ & $\begin{array}{l}0.4 \\
(0,1.0)\end{array}$ & $\begin{array}{l}1.8 \\
(1.6,2.9)\end{array}$ & $\begin{array}{l}6.8 \\
(5.3,8.0)\end{array}$ & $\begin{array}{l}13.3 \\
(12.2,14.8)\end{array}$ & $\begin{array}{l}16.7 \\
(15.2,17.2)\end{array}$ \\
\hline $\begin{array}{l}\text { Tamsulosin non-responders } \\
\text { switching to CT at } 3 \text { months }\end{array}$ & $\begin{array}{l}0 \\
(0,0.4)\end{array}$ & $\begin{array}{l}0.2 \\
(0,0.5)\end{array}$ & $\begin{array}{l}1.3 \\
(1.0,2.3)\end{array}$ & $\begin{array}{l}6.3 \\
(5.0,8.0)\end{array}$ & $\begin{array}{l}13.6 \\
(11.5,15.4)\end{array}$ & $\begin{array}{l}16.2 \\
(14.6,18.4)\end{array}$ \\
\hline $\begin{array}{l}\text { Tamsulosin non-responders } \\
\text { switching to CT at } 6 \text { months }\end{array}$ & $\begin{array}{l}0 \\
(0,0)\end{array}$ & $\begin{array}{l}0.3 \\
(0,0.7)\end{array}$ & $\begin{array}{l}0.9 \\
(0.8,1.9)\end{array}$ & $\begin{array}{l}4.3 \\
(3.0,4.9)\end{array}$ & $\begin{array}{l}9.3 \\
(8.6,10.7)\end{array}$ & $\begin{array}{l}11.6 \\
(10.8,12.9)\end{array}$ \\
\hline $\begin{array}{l}\text { Tamsulosin non-responders } \\
\text { switching to CT at } 12 \text { months }\end{array}$ & $\begin{array}{l}0 \\
(0,0.2)\end{array}$ & $\begin{array}{l}0.4 \\
(0.2,0.6)\end{array}$ & $\begin{array}{l}1.0 \\
(0.8,1.2)\end{array}$ & $\begin{array}{l}3.4 \\
(2.2,4.4)\end{array}$ & $\begin{array}{l}9.4 \\
(7.1,10.1)\end{array}$ & $\begin{array}{l}12.6 \\
(9.7,13.1)\end{array}$ \\
\hline $\begin{array}{l}\text { Tamsulosin non-responders } \\
\text { switching to CT at } 24 \text { months }\end{array}$ & $\begin{array}{l}0 \\
(0,0.1)\end{array}$ & $\begin{array}{l}0.3 \\
(0.2,0.6)\end{array}$ & $\begin{array}{l}1.2 \\
(0.8,1.7)\end{array}$ & $\begin{array}{l}3.0 \\
(2.6,4.0)\end{array}$ & $\begin{array}{l}7.1 \\
(6.4,8.2)\end{array}$ & $\begin{array}{l}10.6 \\
(9.8,12.0)\end{array}$ \\
\hline
\end{tabular}


Table 1 (continued)

\begin{tabular}{|c|c|c|c|c|}
\hline \multirow[t]{2}{*}{ (D) IPSS improvement } & \multicolumn{4}{|c|}{ Percentage of patients (CI) showing improvement at month 48} \\
\hline & $\geq 25 \%$ & $\geq 35 \%$ & $\geq 50 \%$ & $\geq 75 \%$ \\
\hline Combination therapy (CT) & $79.7(78.6,81.2)$ & $73.1(71.6,74.8)$ & $60.8(59.0,62.4)$ & $31.2(27.4,32.6)$ \\
\hline Tamsulosin & $65.5(63.0,68.0)$ & $57.4(55.6,60.0)$ & $44.0(42.5,46.5)$ & $17.0(15.2,18.6)$ \\
\hline Tamsulosin non-responders switching to CT at 1 month & $79.5(76.5,81.4)$ & $71.1(69.3,74.7)$ & $59.3(56.6,60.9)$ & $29.1(27.2,29.9)$ \\
\hline Tamsulosin non-responders switching to CT at 3 months & $78.1(76.8,81.0)$ & $71.0(69.9,73.9)$ & $59.9(56.3,61.2)$ & $28.4(25.8,31.3)$ \\
\hline Tamsulosin non-responders switching to CT at 6 months & $74.1(72.3,75.5)$ & $65.8(64.1,68.6)$ & $52.7(50.6,55.6)$ & $22.4(20.3,24.2)$ \\
\hline Tamsulosin non-responders switching to CT at 12 months & $70.3(67.2,72.1)$ & $62.4(58.7,64.6)$ & $48.4(45.1,51.9)$ & $20.2(18.1,24.4)$ \\
\hline Tamsulosin non-responders switching to CT at 24 months & $71.0(68.5,72.1)$ & $63.2(61.3,65.0)$ & $49.6(46.9,52.2)$ & $19.4(18.0,21.0)$ \\
\hline
\end{tabular}

Panel (A): overview of the patient population transitioning from tamsulosin to tamsulosin-dutasteride combination therapy due to nonresponse to tamsulosin. Panel (B): primary endpoint, i.e. proportion of responders (response rate) and IPSS values at 48 months in patients responding to treatment. The difference in the proportion of responders in each virtual treatment arm relative to the combination therapy arm [Responders $\mathrm{CT}_{\mathrm{CT}}-\operatorname{Responders}_{x}(\%)$ ] summarises the impact of immediate combination therapy. Panel (C): cumulative percentage of subjects switching from moderate or severe to mild symptom scores at each visit. Panel (D): Impact of immediate versus delayed start of tamsulosindutasteride combination therapy on the magnitude of response, as assessed by the proportion of patients showing changes in IPSS $\geq 35 \%, \geq 50 \%$ and $\geq 75 \%$ relative to baseline at month 48 . The statistical significance of the differences between treatment arms for each response threshold is shown for a single replicate trial in Table S5 (see Supplemental Materials)

The results presented above refer to a CTS scenario including placebo effect only after the initial treatment phase. Placebo effect is an important component of the initial response and can last more than 6 months, as assessed by its half-life. No studies included a placebo treatment arm for $>2$ years, so it was not possible to establish whether inter-individual differences might allow for a longer placebo effect. Unless indicated otherwise, values represent median $(90 \%$ CIs) from ten trial replicates. Symptom severity: mild =IPSS 1-7, moderate $=$ IPSS $8-19$, and severe $=$ IPSS $\geq 20$

CI confidence interval, CTS clinical trial simulation, IPSS International Prostate Symptom Score

*Log rank test: $p<0.01$

${ }^{a}$ Baseline IPSS [range] in each treatment arm

${ }^{b}$ percentage of responders (IPSS drop $\geq 25 \%$ relative to baseline) at 48 months

\section{Effect of disease progression, baseline covariate factors and drug treatment on individual IPSS trajectories}

Notwithstanding the body of evidence regarding the benefits of tamsulosin-dutasteride combination therapy, including greater and more durable improvement than with either monotherapy $[14,18,19]$, little attention has been given to the impact of variable rates of disease progression on treatment response or deterioration of symptoms, as measured by IPSS [10, 20, 21]. There are currently no reliable biomarkers that allow identification and prediction of a specific clinical phenotype for disease progression in individual patients, although serum PSA has been explored in this capacity [8, 9]. This is further compounded by limited understanding of the effects of specific comorbidities or other covariates on overall treatment response [22]. Our analysis suggests these limitations may partly be overcome by further characterisation of individual IPSS trajectories.

The introduction of IPSS as a tool for clinical practice and in research protocols was originally based on data from relatively short-term validation steps [23]. Among the available reports on the natural history of LUTS, long-term longitudinal follow-up studies have been restricted to changes in IPSS relative to baseline, making it difficult to distinguish the impact of multiple interacting factors on the deterioration of symptoms. Exploratory investigations have suggested that IPSS does not correlate with laboratory measures of urinary function at baseline (e.g., PV and PSA), but limitations in the methodology employed indicate that this should not be taken as conclusive [24, 25]. In fact, the lack of such correlations has been often assigned to study design, inclusion and exclusion criteria, and sample size. Nevertheless, baseline IPSS has been shown to predict the risk of progression in several prior clinical trials [6-8].

These apparently paradoxical findings may reflect the empirical nature of past research protocols, which assess or infer disease progression directly from the observed increase in IPSS over time, rather than based on a specific parameter that captures the underlying processes [26-28]. In this context, the initial simulations show the implications of the interaction between baseline covariates (e.g. IPSS) and varying rates of disease progression on individual trajectories (see Supplemental Materials). Although the possibility that patients with LUTS/BPH may show intrinsic differences in response to $\alpha$-blockers and 5ARI cannot be excluded, it is clear from figures $\mathrm{S} 1, \mathrm{~S} 2$ and $\mathrm{S} 3$ that the identification of inter-individual variation in progression rates only from 
baseline characteristics may be challenging. Further exploration is required to identify whether baseline markers may explain some of this heterogeneity in symptom deterioration and consequently in treatment response.

\section{Clinical trial simulations: impact of immediate versus delayed start of combination therapy}

While the use of combination therapy in LUTS/BPH patients with moderate and severe symptoms at risk of disease progression is endorsed by international guidelines, this analysis was performed taking into account prior evidence of the favourable benefit-risk profile of dutasteride-tamsulosin combination [29]. An early start of treatment with combination therapy should not alter the overall safety profile. However, the current results show that symptomatic treatment does not stop disease progression, and potentially reduces the benefits associated with the 5-ARI disease-modifying properties, if treatment is delayed for more than 6 months.

To address the key research question of this investigation, we have focused on the results of a parallel-study design, which reflects a typical clinical trial setting in which patients are randomised to different treatment arms, even though it does not fully capture the implications of delayed start of combination therapy for every single patient. In this regard, our results do not exclude the contribution of betweenpatient variability on predicted treatment response. It is worth mentioning that baseline characteristics among tamsulosin non-responders do not differ significantly from patients on combination therapy raising questions as to whether baseline demographics and clinical features may be sufficiently discriminative in determining treatment response. Yet, early administration of tamsulosin-dutasteride combination therapy (i.e. <6-month delay) not only results in a significantly greater responder rate at month 48 in men at risk of progression, but also leads to an increase in the fraction of patients transitioning to lower IPSS severity levels. More strikingly, early use of combination therapy results in a larger proportion of patients having greater clinical improvement, i.e. changes in IPSS relative to baseline (Table 1, panels $\mathrm{C}$ and D). Early start of combination treatment allows $\sim 10 \%$ more patients to benefit from symptomatic improvement.

The clinical relevance of differences in IPSS may be questioned without careful understanding of the approach used here. First, the definition of response based on $a \geq 25 \%$ reduction in IPSS relative to baseline allows for a standardised measure across the entire population, irrespective of symptom severity at baseline, provided that the response strongly influences the relationship between the perception of improvement and the score improvement. This threshold of $\geq 25 \%$ reduction in IPSS has been used as a study endpoint in various clinical trials included in this CTS [14,
15]. Second, CTS were performed without residual noise to ensure accurate estimates of the effect of the different interventions. Residual noise reduces the sensitivity to detect a true treatment response.

Even though a few assumptions are required to assess treatment response when designing a standard clinical trial protocol or a virtual one, as in our case, our results highlight that immediate exposure to tamsulosin-dutasteride combination therapy can have a long-lasting impact on the individual trajectories of a small, but clinically relevant fraction of patients (i.e. those who have a faster disease progression rate but cannot be identified at start of treatment). Considering the chronic nature of the disease, the effect of diseasemodifying properties cannot be compensated by symptomatic interventions over the longer term.

Undeniably, there are limitations in the work performed. First, it should be recognised that the population used for the development of the longitudinal model represents a subgroup of the overall BPH patient population, and as such encompasses only subjects with moderate or severe symptoms at risk of progression. In addition, we have assumed that the placebo effect starts at the beginning of the trial and not when the therapy is switched at later time points. As the longitudinal model used for the simulations showed some bias in terms of the predicted trajectories severe patients with very high IPSS scores (i.e., upper 2.5 th percentile), there is a chance of misclassification of non-responders. Last, we have not evaluated IPSS profiles beyond 48 months to ensure that simulation results could be supported by existing clinical data, i.e., the time span used in the analysis matches the duration of the longest clinical trial included in the development of the model.

\section{Conclusions}

We demonstrated how CTS can be used to describe individual IPSS trajectories and explore the implications of immediate versus delayed start of tamsulosin-dutasteride combination therapy in men at risk of disease progression. Importantly, simulation scenarios showed higher overall response rates and significantly lower IPSS values following immediate combination therapy. Initiation of monotherapy in LUTS/BPH patients with moderate or severe symptoms, who are at risk of disease progression, may offer less advantage; it appears to reduce the long-term benefit from early combination therapy. Patients eligible to initiate combination therapy may miss the symptomatic benefit over the long term, as shown by the significant difference in proportion of responders was observed in the treatment arms, when switching from tamsulosin occurs at 6 months or later. 
Acknowledgements Medical writing support was provided by Lisa Auker, PhD of Fishawack Indicia Ltd, UK, and funded by GSK in accordance with Good Publication Practice (GPP3) guidelines (http:// www.ismpp.org/gpp3).

Author contributions SD was involved in the conception/design of the study, analysis and interpretation of study data, drafting and critical revision of the manuscript, and approved its submission. TW was involved in the conception/design of the study, acquisition and interpretation of study data, drafting and critical revision of the manuscript, and approved its submission. BA was involved in the interpretation of study data, drafting and critical revision of the manuscript, and approved its submission. MM was involved in the conception of the study and interpretation of study data, drafting and critical revision of the manuscript, and approved its submission. JMPM was involved in the conception of the study and interpretation of study data, drafting and critical revision of the manuscript, and approved its submission. $\mathrm{CC}$ was involved in the interpretation of study data, drafting and critical revision of the manuscript, and approved its submission. MO was involved in the interpretation of study data, drafting and critical revision of the manuscript, and approved its submission. CR was involved in the interpretation of study data, drafting and critical revision of the manuscript, and approved its submission. ODP was involved in the conception/design and interpretation of study data, drafting and critical revision of the manuscript, and approved its submission.

Funding This investigation was funded by GlaxoSmithKline (GSK). Anonymised individual participant data and study documents can be requested for further research from www.clinicalstudydatarequest.com.

\section{Compliance with ethical standards}

Conflict of interest SD has none to declare. TW holds stocks/shares in GSK. BA, MM, JMPM, CC and ODP are GSK employees and hold stocks/shares in GSK. MO has been a speaker, consultant and/or trial participant for Apogepha, Astellas, Duchesnay, Ferring, GSK, Lilly, Pierre Fabre and Pfizer, and received research grants from Astellas and Pfizer. CR was previously employed as a consultant to GSK.

Open Access This article is distributed under the terms of the Creative Commons Attribution 4.0 International License (http://creativeco mmons.org/licenses/by/4.0/), which permits unrestricted use, distribution, and reproduction in any medium, provided you give appropriate credit to the original author(s) and the source, provide a link to the Creative Commons license, and indicate if changes were made.

\section{References}

1. Emberton M, Cornel EB, Bassi PF et al (2008) Benign prostatic hyperplasia as a progressive disease: a guide to the risk factors and options for medical management. Int J Clin Pract 62:1076-1086

2. McVary KT, Roehrborn CG, Avins AL et al (2011) Update on AUA guideline on the management of benign prostatic hyperplasia. J Urol 185:1793-1803

3. O'Leary MP (2000) LUTS, ED, QOL: alphabet soup or real concerns to aging men? Urology 56:7-11

4. Gravas S, Cornu JN, Drake MJ et al (2018) EAU guidelines on the management of non-neurogenic male lower urinary tract symptoms (LUTS), incl. benign prostatic obstruction (BPO). Available at: http://uroweb.org/guideline/treatment-of-non-neurogenic -male-luts/. Accessed 5 May 2019
5. Debruyne F, Barkin J, van Erps P et al (2004) Efficacy and safety of long-term treatment with the dual 5 alpha-reductase inhibitor dutasteride in men with symptomatic benign prostatic hyperplasia. Eur Urol 46:488-494 (discussion 95)

6. McConnell JD, Roehrborn CG, Bautista OM et al (2003) The long-term effect of doxazosin, finasteride, and combination therapy on the clinical progression of benign prostatic hyperplasia. $\mathrm{N}$ Engl J Med 349:2387-2398

7. Roehrborn CG, Barkin J, Siami P et al (2011) Clinical outcomes after combined therapy with dutasteride plus tamsulosin or either monotherapy in men with benign prostatic hyperplasia (BPH) by baseline characteristics: 4-year results from the randomized, double-blind Combination of Avodart and Tamsulosin (CombAT) trial. BJU Int 107:946-954

8. Kozminski MA, Wei JT, Nelson J, Kent DM (2015) Baseline characteristics predict risk of progression and response to combined medical therapy for benign prostatic hyperplasia (BPH). BJU Int 115:308-316

9. Pinto F, Racioppi M, Sacco E et al (2009) Progression, risk factors and subsequent medical management of symptomatic benign prostatic hyperplasia. Arch Ital Urol Androl 81:1-8

10. Fitzpatrick JM (2006) The natural history of benign prostatic hyperplasia. BJU Int 97(Suppl 2):3-6 (discussion 21-2)

11. Chan PL, Holford NH (2001) Drug treatment effects on disease progression. Annu Rev Pharmacol Toxicol 41:625-659

12. Jonsson F, Marshall S, Krams M, Jonsson EN (2005) A longitudinal model for non-monotonic clinical assessment scale data. J Pharmacokinet Pharmacodyn 32:795-815

13. D'Agate S, Wilson T, Adalig B, et al. (2018) Development of a drug-disease model describing individual IPSS trajectories in BPH patients: implication of disease progression and covariate factors on long term treatment response. In: annual meeting of the Population Approach Group in Europe (PAGE), Montreux, Switzerland. PAGE 27 Abstr 8794 [www.page-meeti ng.org/?abstract=8794]

14. Roehrborn CG, Siami P, Barkin J et al (2010) The effects of combination therapy with dutasteride and tamsulosin on clinical outcomes in men with symptomatic benign prostatic hyperplasia: 4-year results from the CombAT study. Eur Urol 57:123-131

15. Roehrborn CG, Oyarzabal Perez I, Roos EP et al (2015) Efficacy and safety of a fixed-dose combination of dutasteride and tamsulosin treatment (Duodart ${ }^{\circledR}$ ) compared with watchful waiting with initiation of tamsulosin therapy if symptoms do not improve, both provided with lifestyle advice, in the management of treatmentnaive men with moderately symptomatic benign prostatic hyperplasia: 2-year CONDUCT study results. BJU Int 116:450-459

16. Della Pasqua O (2011) PKPD and disease modelling: concepts and applications to oncology. In: Kimko HHC, Peck CC (eds) AAPS Advances in the pharmaceutical sciences series, vol 1. Springer, New York, pp 277-306

17. Santen G, Horrigan J, Danhof M, Della Pasqua O (2009) From trial and error to trial simulation. Part 2: an appraisal of current beliefs in the design and analysis of clinical trials for antidepressant drugs. Clin Pharmacol Ther 86:255-262

18. Dimitropoulos K, Gravas S (2016) Fixed-dose combination therapy with dutasteride and tamsulosin in the management of benign prostatic hyperplasia. Ther Adv Urol 8:19-28

19. Gravas S, Oelke M (2010) Current status of 5alpha-reductase inhibitors in the management of lower urinary tract symptoms and BPH. World J Urol 28:9-15

20. Fujimura T, Kume H, Nishimatsu H et al (2012) Assessment of lower urinary tract symptoms in men by international prostate symptom score and core lower urinary tract symptom score. BJU Int 109:1512-1516 
21. Glass AS, Hilton JF, Cowan JE, Washington SL, Carroll PR (2014) Serial prostate biopsy and risk of lower urinary tract symptoms: results from a large, single-institution active surveillance cohort. Urology 83:33-38

22. Strand DW, Costa DN, Francis F, Ricke WA, Roehrborn CG (2017) Targeting phenotypic heterogeneity in benign prostatic hyperplasia. Differentiation 96:49-61

23. Barry MJ, Fowler FJ Jr, O'Leary MP et al (1992) The American Urological Association symptom index for benign prostatic hyperplasia. The measurement committee of the American Urological Association. J Urol 148:1549-1557 (discussion 64)

24. Fukuta F, Masumori N, Mori M, Tsukamoto T (2012) Natural history of lower urinary tract symptoms in Japanese men from a 15-year longitudinal community-based study. BJU Int 110:1023-1029

25. Tsukamoto T, Masumori N, Rahman M, Crane MM (2007) Change in International Prostate Symptom Score, prostrate-specific antigen and prostate volume in patients with benign prostatic hyperplasia followed longitudinally. Int J Urol 14:321-324
26. Holford NH, Chan PL, Nutt JG et al (2006) Disease progression and pharmacodynamics in Parkinson disease-evidence for functional protection with levodopa and other treatments. J Pharmacokinet Pharmacodyn 33:281-311

27. Mould D (2007) Developing models of disease progression. In: Ette E, Williams PJ (eds) Pharmacometrics: the science of quantitative pharmacology. Wiley, Hoboken, pp 547-581

28. Lepor H (1998) Phase III multicenter placebo-controlled study of tamsulosin in benign prostatic hyperplasia. Tamsulosin Investig Group. Urol 51:892-900

29. Duodart European approved summary of product characteristics. Last update 23/11/2017. Online accessible through https://mri.ctsmrp.eu/Human/Downloads/DE_H_2251_001_FinalSPC_1of7. pdf. Accessed April 2019

Publisher's Note Springer Nature remains neutral with regard to jurisdictional claims in published maps and institutional affiliations. 